\title{
Tool Morphology Constrains the Effects of Tool Use on Body Representations
}

\author{
Luke E. Miller \\ University of California, San Diego
}

\author{
Matthew R. Longo \\ Birkbeck, University of London
}

\author{
Ayse P. Saygin \\ University of California, San Diego
}

\begin{abstract}
What factors constrain whether tool use modulates the user's body representations? To date, studies on representational plasticity following tool use have primarily focused on the act of using the tool. Here, we investigated whether the tool's morphology also serves to constrain plasticity. In 2 experiments, we varied whether the tool was morphologically similar to a target body part (Experiment 1, hand; Experiment 2, arm). Participants judged the tactile distance between pairs of points applied to their tool-using target body surface and forehead (control surface) before and after tool use. We applied touch in 2 orientations, allowing us to quantify how tool use modulates the representation's shape. Significant representational plasticity in hand shape (increase in width, decrease in length) was found when the tool was morphologically similar to a hand (Experiment 1A), but not when the tool was arm-shaped (Experiment 1B). Conversely, significant representational plasticity was found on the arm when the tool was arm-shaped (Experiment 2B), but not when hand-shaped (Experiment 2A). Taken together, our results indicate that morphological similarity between the tool and the effector constrains tool-induced representational plasticity. The embodiment of tools may thus depend on a match-to-template process between tool morphology and representation of the body.
\end{abstract}

Keywords: tool, embodiment, body representation, touch, perception

Tool use is one of the hallmark features of the human species. Tools are used in almost every facet of our lives, from the most mundane (e.g., knives for slicing food) to the most awe-inspiring (e.g., robotic surgical tools). Tools can help overcome the limitations of our bodies, changing the way we interact with and manipulate the environment. However, the influence of tool use is not limited to the way the body is used; tools can also change the way we represent our own body.

This article was published Online First August 25, 2014.

Luke E. Miller, Department of Cognitive Science and Kavli Institute for Brain and Mind, University of California, San Diego; Matthew R. Longo, Department of Psychological Sciences, Birkbeck, University of London; Ayse P. Saygin, Department of Cognitive Science and Kavli Institute for Brain and Mind, University of California, San Diego.

We would like to thank Kavli Institute for Brain and Mind, University of California, San Diego (UCSD), for supporting this research. Luke E. Miller was additionally supported by an National Institute of Mental Health training grant from the Institute for Neural Computation, UCSD; Ayse P. Saygin by National Science Foundation (CAREER BCS1151805) and Defense Advanced Research Project Agency; and Matthew R. Longo by the Royal Society, Experimental Psychology Society, and European Research Council. We thank Alessandro Farnè and two anonymous reviewers for their helpful comments, Paola Rossi for help collecting data, and Ivan Owen for constructing the hand-shaped tool.

Correspondence concerning this article should be addressed to Luke E. Miller, 9500 Gilman Drive, Department of Cognitive Science, University of California San Diego, La Jolla, CA, 92093-0515. E-mail: lumiller@ucsd.edu
The idea that the brain treats a tool as part of the body has been around for over a century (Butler, 1872). More recent empirical support for this claim has come from neuroscience and experimental psychology (Maravita \& Iriki, 2004). Using a tool leads to plastic changes in the user's body representations (i.e., representational plasticity). Studies with macaque monkeys (Iriki, Tanaka, \& Iwamura, 1996) and humans (Farnè, Iriki, \& Làdavas, 2005; Maravita, Spence, Kennett, \& Driver, 2002) have demonstrated that the neural representation of the space around the hand extends to include a tool after use. Tool use can also modulate representations of the body itself, as evidenced by changes in reaching kinematics (Cardinali, Frassinetti, et al., 2009), distal shifts in localization of touch on the arm wielding the tool (Cardinali et al., 2011), and altered perception of the midpoint of the arm (Sposito, Bolognini, Vallar, \& Maravita, 2012). Together, these results indicate that tool use has widespread influences on the representation of not only the peripersonal space surrounding the body but also the body itself.

What factors constrain representational plasticity following tool use? One proposal that has received considerable attention is that plasticity is driven by the functional consequences of tool use. Indeed, representational plasticity has been found following active use (Bonifazi, Farnè, Rinaldesi, \& Làdavas, 2007; Farnè et al., 2005; Farnè \& Làdavas, 2000; Maravita, Clarke, Husain, \& Driver, 2002; Maravita, Spence, et al., 2002; Serino, Bassolino, Farnè, \& Làdavas, 2007), and in preparation to use the tool (Costantini, Ambrosini, Sinigaglia, \& Gallese, 2011; Holmes, Calvert, \& Spence, 2007; Witt, Proffitt, \& Epstein, 2005), but not when the 
tool was passively held (Kao \& Goodale, 2009; Maravita, Spence, et al., 2002).

Another potential factor constraining plasticity-one that has yet to receive attention from researchers-is the morphology of the tool. Tools do not need to be shaped like the body in order to be effective. On the contrary, the efficacy of some tools (e.g., corkscrews or knives) is critically dependent on the very fact that they have morphological features very different from our bodies. Nevertheless, the embodiment of an external object can be contingent upon it having a similar overall form (i.e., size and shape) to one's own body. For example, the experience of illusory body ownership of an object in the rubber hand illusion (RHI) is dependent on that object having the overall shape of a hand (Haans, IJsselsteijn, \& de Kort, 2008; Tsakiris, Carpenter, James, \& Fotopoulou, 2010; Tsakiris, Costantini, \& Haggard, 2008; Tsakiris \& Haggard, 2005).

Although morphology constrains conscious feelings of embodiment, such as the sense of limb ownership, whether or not morphological constraints regulate plasticity induced by tool use is currently unknown. Although we are certainly able to use tools that are not shaped like our body or body parts, function is often constrained by morphology. The tight coupling between a tool's shape and its usability increases the probability that tools shaped like the body can be used like the body. Here, we hypothesized that tool shape plays a role in modulating plasticity. In other words, we explored whether tool-induced representational plasticity of a body part is constrained by the tool's morphological resemblance to that body part. We tested the role of tool morphology in representational plasticity using a tactile distance judgment (TDJ) task, which has been used in previous studies to measure plasticity of body representations (de Vignemont, Ehrsson, \& Haggard, 2005; Tajadura-Jiménez et al., 2012; Taylor-Clarke, Jacobsen, \& Haggard, 2004). Unlike most previous studies (though see Canzoneri et al., 2013), we administered the TDJ in two orientations: proximodistally (i.e., along the width of the hand) and mediolaterally (i.e., across the length of the hand). This allowed us to explore whether any representational plasticity we observe would be manifest as an overall size change or a shape change.

Perceiving the size of objects touching the skin depends on an implicit representation of body form (Longo, Azanon, \& Haggard, 2010). Accordingly, if tool use leads to representational plasticity, we would expect to see changes in the perceived size of objects contacting the skin surface. We measured changes in tactile size perception on the hand and arm in four experiments manipulating the morphological similarity between the tool and the effector. The tools used in each experiment differed in their morphology (handshaped or arm-shaped), but not in their functional "goal" (both tools were used to grasp and move objects). We predicted that the hand-shaped tool would lead to greater modulation of the implicit representation of the hand, whereas the arm-shaped tool would lead to greater modulation of the implicit representation of the arm.

\section{Method}

\section{Participants}

Fifteen adults (14 female) between 18 and 27 years of age (mean $=21.01, S D=2.03)$ participated in Experiment 1A. Fifteen adults (13 female) between 19 and 27 years of age (mean $=21.58$, $S D=1.99)$ participated in Experiment 1B. Fourteen participants
(13 female) between 18 and 34 years of age $($ mean $=21.15, S D=$ 3.96) participated in Experiment 2A. Ten participants (6 female) between 18 and 25 years of age (mean $=21.47, S D=2.31)$ participated in Experiment 2B. No participant took part in more than one experiment. All participants were right-handed, as indicated by self-report. The experiments were approved by the University of California, San Diego, ethics committee and all participants gave informed consent.

\section{Materials}

In Experiments $1 \mathrm{~A}$ and $2 \mathrm{~A}$, participants wore a custom-built plastic hand-shaped tool, which was modeled after a human hand (Figure 1A). Each finger of the tool contained biologically realistic, fully adjustable "joints." The user's fingers and those of the tool were connected via straps that allowed for control of each of the tool's fingers individually. Thus, the fingers of the handshaped tool were contingent upon the movement of the user's actual fingers and resembled the user's fingers in their dexterity. The tool was approximately $21 \mathrm{~cm}$ wide, as measured from the base of the pointer finger to the base of the pinky, and $45 \mathrm{~cm}$ long, as measured from the base of the tool to the tip of the middle finger. In Experiments 1B and 2B, participants used an 80-cm-long arm-shaped mechanical grabber (Figure 1B). The grabber consisted of a handle, a long slender shaft, and pincers at its distal tip. In contrast to the hand-shaped tool, the movement of the grabber's pincers did not retain the dexterity of a human hand. To grasp an object with the grabber, participants squeezed a vertical handle in order to horizontally close pincers at its distal tip. The movement profile of the grabber's shaft, however, did resemble the user's arm during movement. In both experiments, participants used their assigned tool to pick up and move balloons.

In the TDJ task, touch was delivered using wooden posts, each mounted on a square wooden block, and separated by three distances: 20, 30, and $40 \mathrm{~mm}$. Each post was tapered to a flat point 1 $\mathrm{mm}$ in diameter.
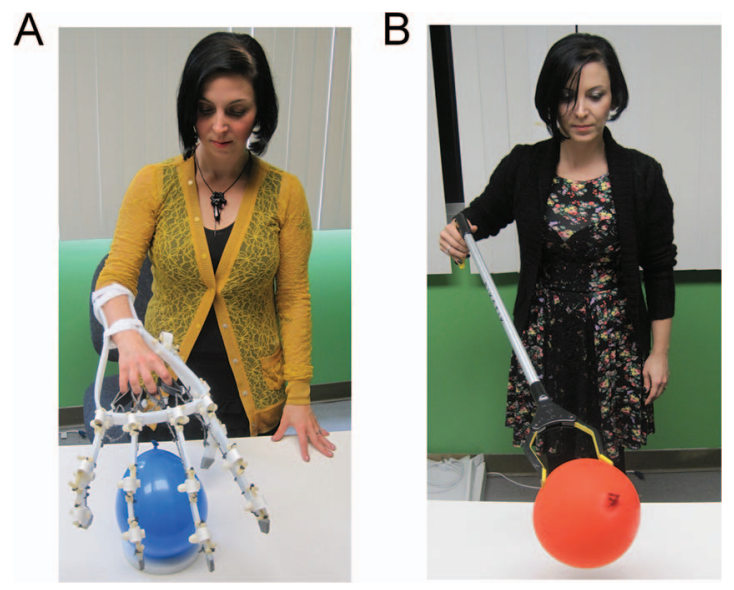

Figure 1. Tools used in the experiments. (A) Hand-shaped tool used in Experiments 1A and 2A. (B) Mechanical grabber used in Experiments $1 \mathrm{~B}$ and $2 \mathrm{~B}$. The color version of this figure appears in the online article only. 


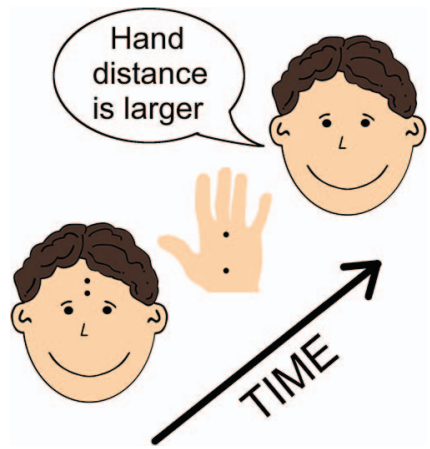

Figure 2. Tactile distance judgment task. Depicted is a typical trial of the tactile distance judgment task. In this case, the target body part is the hand, and the control surface is the forehead. On this trial, the participant is first presented with two tactile points along the forehead, and then, after approximately $2 \mathrm{~s}$, with two points along the hand. The participant then makes a verbal judgment about which body part was touched with a greater distance. On this trial, the participant judged the two tactile points on the hand to be further apart than on the head, consistent with the actual physical difference. The color version of this figure appears in the online article only.

\section{Procedure}

The experiments began with a pre-tool use TDJ, followed by tool use, and a post-tool-use TDJ. In the TDJ (see Figure 2), touch was administered to a target and reference surface. In Experiments $1 \mathrm{~A}$ and $2 \mathrm{~A}$, the target surface was the dorsum of the hand, whereas it was the dorsum of the forearm in Experiments $1 \mathrm{~B}$ and $2 \mathrm{~B}$. The reference surface was the forehead in all experiments. Participants made unspeeded two-alternative forced-choice verbal judgments about which body surface was stimulated with the greatest tactile distance ("hand/arm" or "forehead"). One of five distance pairs was used to administer touch to the forehead and hand/arm on each trial (target/reference): $40 / 20 \mathrm{~mm}, 30 / 20 \mathrm{~mm}, 30 / 30 \mathrm{~mm}, 20 / 30$ $\mathrm{mm}, 20 / 40 \mathrm{~mm}$. Tactile stimulation in each trial occurred in one of two orientations: across $($ hand $/ \mathrm{arm}=$ mediolateral; forehead $=$ eye-to-eye) or along (hand/arm = proximodistal; forehead = nasion to hairline) the body surface. Each distance pair was applied 8 times in each orientation, yielding a total of 80 trials, 40 in each orientation. The body part that was stimulated first (target or reference) was counterbalanced for each distance combination and orientation. The procedure was split into two blocks (40 trials each), separated by an 8-min break in the pre-tool-use condition or $8 \mathrm{~min}$ of tool use in the post-tool-use condition. On each trial, the distance combination, stimulus orientation, and order in which body surfaces were stimulated were selected from a randomized list of all possible stimulation combinations for that block. Tactile stimuli were applied manually by the experimenter, and lasted roughly $1 \mathrm{~s}$, with an approximately 2 -s interstimulus interval when switching between surfaces.

Immediately following the pre-tool-use TDJ, participants were asked to use the tool. They were instructed to pick up a balloon and place it into a bucket repeatedly. The balloon was initially positioned approximately at the subject's midline and approximately $75 \mathrm{~cm}$ from their body in Experiments $1 \mathrm{~A}$ and 2A, and approximately $110 \mathrm{~cm}$ in Experiments $1 \mathrm{~B}$ and $2 \mathrm{~B}$; the bucket was placed approximately $75 \mathrm{~cm}$ to the right of the subject's midline. Once the balloon was placed inside the bucket by the subject, the experimenter removed it and placed it back into position. Three differently sized balloons were used during the course of the task and were alternated to keep the task engaging. The grasping task for the two tools varied slightly because of the differences in their dexterity. The task for the hand-shaped tool was as follows: For the 3 min, subjects picked up the balloons using a power grip. For 1 min each, the subjects first picked up the largest, then medium, and then smallest balloon. Subjects then picked up the smallest balloon using a precision grip with each thumb-finger combination (thumb-pointer, thumb-middle, thumb-ring, thumb-pinky) for 1 min each, totaling $4 \mathrm{~min}$. For the final minute, subjects again used a power grip to pick up the smallest balloon. The task for the mechanical grabber was as follows: For the first $3 \mathrm{~min}$, subjects picked up the balloons with the grabber's pincers. For 1 min each, the subjects first picked up the largest, then medium, and then smallest balloon. Subjects then spent the next 4 min alternating between picking up the medium-sized and smallest balloons. The final minute was spent picking up the smallest balloon. This task was self-paced, and was done twice, each instance lasting approximately $8 \mathrm{~min}$, for a total of $16 \mathrm{~min}$ of tool use during the course of each experiment.

\section{Analysis Methods}

Psychophysical curve fitting was used to measure changes in tactile size perception on the hand following tool use. The curvefitting procedure and all corresponding analyses were performed with MATLAB (The MathWorks, Natick, MA) using the Palamedes Toolbox (Prins \& Kingdom, 2009). Logistic functions were fit to each participant's pre- and post-tool-use response profiles using a maximum likelihood procedure (Wichmann \& Hill, 2001a). We then extracted from each curve the point of subjective equality (PSE; the point on the psychometric curve that crosses $50 \%$, and indicates the point at which the two stimuli would be perceived as equal) and the just-noticeable difference (JND). In this context, the PSE is a measure of anisotropy in tactile size perception, whereas the JND is a measure of discriminative sensitivity. We used changes to the PSE and JND in each dimension as dependent measures in repeated measures ANOVAs to evaluate the effects of tool use.

In addition to statistical analyses using dependent measures derived from the psychometric curves, we also compared the group-level curves for pre- and post-tool-use TDJ directly using a permutation test called a likelihood ratio test (LRT; Wichmann \& Hill, 2001b). $P$ values for the LRT correspond to the ratio of the simulated data sets that had larger likelihood values than the true data. Each LRT was based on 5,000 simulations. If the number of simulated likelihood values greater than the true data exceeded 250 , the pre- and post-tool-use curves were not considered significantly different (i.e., $p>.05$ ).

\section{Results}

We depict the results first as psychophysical curves for pre- and post-tool-use data (Figures 3 and 4), followed by comparisons within and across experiments (Figure 5). 


\section{Experiment 1A: Hand-Shaped Tool and Tactile Perception on the Hand}

Significant modulations of tactile size perception on the hand were found following the use of the hand-shaped tool. Tool use changed tactile size perception on the hand in both orientations (Figure 3; Figure 5A). A 2 (Tool Use: Pre, Post) $\times 2$ (Orientation: Across, Along) repeated-measures analysis of variance (ANOVA) with PSE as the dependent measure revealed main effects of tool use, $F(1,14)=4.73, p=.047, \eta_{\mathrm{p}}^{2}=0.25$, and orientation, $F(1$, $14)=8.83, p=.01, \eta_{\mathrm{p}}^{2}=0.38$. We also found a highly significant interaction between the two factors, $F(1,14)=48.88, p<.0001$, $\eta_{\mathrm{p}}^{2}=0.78$, which was driven by opposing changes in tactile size perception on the hand for each orientation. Tool use led to an increase in tactile size perception across the hand ( $8.9 \%$ increase), $t(14)=3.64, p=.003, \eta^{2}=0.49$, and a decrease along the hand (17.3\% decrease), $t(14)=-7.53, p<.001, \eta^{2}=0.80$.

Tool use did not change the discriminative sensitivity on the hand as measured by the JND. A 2 (Tool Use: Pre, Post) $\times 2$ (Orientation: Across, Along) repeated-measures ANOVA revealed a main effect of orientation, $F(1,14)=5.34, p=.037, \eta_{p}^{2}=0.28$, likely reflecting preexisting differences in the sensitivity on the hand and forehead. No other main effects or interactions were found (all $F \mathrm{~s}<1.75$; all $p \mathrm{~s}>0.2$ ).

Analysis of the group-level psychometric curves also demonstrated analogous results. LRTs demonstrated that tool use significantly changed tactile size perception across $(p=.02)$ and along $(p<.001)$ the hand (see Figure 3). Significant changes in the post-tool-use curves were driven by changes in the PSE (all $p$ s $<$ 0.05 ) and not the JND (all $p$ s $>0.1$ ).

\section{Experiment 1B: Arm-Shaped Tool and Tactile Perception on the Hand}

In striking contrast to the hand-shaped tool, the use of the arm-shaped mechanical grabber did not lead to tool-induced modulations on the hand in either orientation (Figure 5A). A repeated

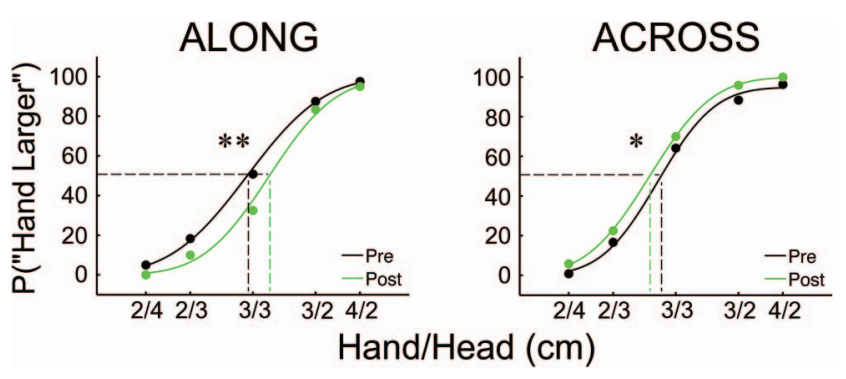

Figure 3. Pre- and post-tool-use curves for Experiment 1A. The pre-tooluse (black) and post-tool-use (gray) curves along (left) and across (right) the hand. The $y$-axis is the probability that the subject responded that touch on the hand felt larger than the forehead for each distance combination ( $x$-axis). Crosshairs in the center of each plot correspond to the point crossing the $50 \%$ mark on the $y$-axis (horizontal line) and the middle distance combination (vertical line). A clear rightward shift in the along post-tool-use curve indicates a compression in the perceived tactile size of stimuli. The leftward shift in the across post-tool-use curve indicates an expansion in the perceived tactile size of stimuli. The color version of this figure appears in the online article only. ${ }^{*} p<0.05 .{ }^{* * *} p<0.01$.

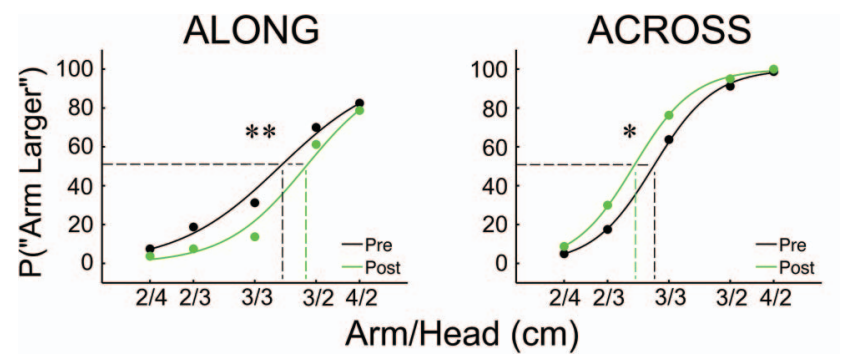

Figure 4. Pre- and post-tool-use curves for Experiment 2B. The pre-tooluse (black) and post-tool-use (gray) curves along (left) and across (right) the hand. The y-axis is the probability that the subject responded that touch on the arm felt larger than the forehead for each distance combination ( $x$-axis). Crosshairs in the center of each plot correspond to the point crossing the $50 \%$ mark on the $y$-axis (horizontal line) and the middle distance combination (vertical line). A clear rightward shift in the along post-tool-use curve indicates a compression in the perceived tactile size of stimuli. The leftward shift in the across post-tool-use curve indicates an expansion in the perceived tactile size of stimuli. The color version of this figure appears in the online article only. ${ }^{*} p<0.05$. ${ }^{* *} p<0.01$.

measures ANOVA on the PSE found no significant interactions or main effects (all $F \mathrm{~s}<0.1$; all $p$ s $>0.7$ ). A repeated measures ANOVA on the JND found no significant main effects or interactions (a trend for a main effect of orientation, $F[1,14]=3.32, p=$ $.09, \eta_{\mathrm{p}}^{2}=0.19$; other $F \mathrm{~s}<0.15$, all $p \mathrm{~s}>0.7$ ). Analysis of the psychometric curves using LRT also found no differences between the pre- and post-tool-use curves (all $p \mathrm{~s}>0.7$ ).

\section{Experiment 1A Versus 1B}

In order to more precisely confirm our hypothesis that tool shape modulates tactile size perception, it is necessary to demonstrate that changes in TDJ following the use of the hand-shaped tool is significantly greater than that for the arm-shaped grabber. We therefore performed a $2 \times 2$ ANOVA with the type of tool used (hand-shaped, arm-shaped) as a between-subjects factor, and the orientation of touch (across, along) as a within-subjects factor, with the dependent measure being the difference between the preand post-tool-use PSEs. Changes in tactile size perception significantly differed based on the type of tool used (Figure 5A). The critical interaction between tool type and orientation was significant, $F(2,28)=17.22, p<.001, \eta_{\mathrm{p}}^{2}=0.38$. Follow-up pairwise comparisons demonstrated a significant difference in size change between the two experiments for TDJ both across, $t(28)=2.40, p=.02, \eta^{2}=$ 0.17 , and along, $t(28)=-3.24, p=.003, \eta^{2}=0.27$, the hand.

\section{Experiment 2A: Hand-Shaped Tool and Tactile Perception on the Arm}

The hand-shaped tool did not lead to significant changes in tactile size perception on the arm in either orientation (Figure 5B). A 2 (Tool Use: Pre, Post) $\times 2$ (Orientation: Across, Along) repeated-measures ANOVA on the PSEs revealed a significant main effect of orientation, $F(1,13)=10.68, p=.006, \eta_{\mathrm{p}}^{2}=0.45$, because of preexisting orientation-specific differences in tactile size perception on the arm. No other main effects or interactions were found (all $F \mathrm{~s}<1$; all $p \mathrm{~s}>0.38$ ]. Similar results were found 
for the JNDs: There was a significant main effect of orientation, $F(1,13)=28.93, p<.0001, \eta_{\mathrm{p}}^{2}=0.69$, and a marginally significant main effect of tool use, $F(1,13)=3.59, p=.08, \eta_{\mathrm{p}}^{2}=$ 0.22 . However, the crucial interaction was not significant, $F(1$, $13)=0.19, p=.67$. Analysis of the psychometric curves using LRTs also found no differences between the pre- and post-tool-use curves (all $p \mathrm{~s}>0.6$ ).

\section{Experiment 2B: Arm-Shaped Tool and Tactile Perception on the Arm}

In striking contrast to Experiment 2A, as well as Experiment 1B, we found significant changes to tactile size perception on the arm following the use of the arm-shaped tool (Figure 4; Figure 5B). Tool use changed tactile size perception on the arm in both orientations. A 2 (Tool Use: Pre, Post) $\times 2$ (Orientation: Across, Along) repeated-measures ANOVA on the PSEs revealed a significant main effect of orientation, $F(1,9)=39.41, p<.0001$, $\eta_{\mathrm{p}}^{2}=0.81$, but no significant main effect of tool use, $F(1,9)=$ $0.42, p=.53$. Like Experiment $1 \mathrm{~A}$, we found a significant interaction between tool use and orientation, $F(1,9)=14.62, p=.004$, $\eta_{\mathrm{p}}^{2}=0.62$, demonstrating opposing changes on the arm for each orientation. Tool use led to an increase in tactile size perception across the arm (16.5\% increase), $t(9)=2.72, p=.024, \eta^{2}=0.45$, and a decrease along the arm (22.5\% decrease), $t(9)=-2.95, p=$ $.016, \eta^{2}=0.49$.

Tool use did not change the discriminative sensitivity on the arm. An ANOVA on the JNDs revealed a significant main effect of orientation, $F(1,9)=6.44, p=.036, \eta_{\mathrm{p}}^{2}=0.42$. No other main effects or interactions were found (all $F \mathrm{~s}<1.5$; all $p \mathrm{~s}>0.25$ ).

Analysis of the group-level psychometric curves also demonstrated that tool use modulated tactile size perception on the arm. LRTs demonstrated that tool use significantly changed tactile size perception across $(p=.018)$ and along $(p<.0001)$ the arm (see Figure 4). Significant changes in the post-tool-use curves were driven by changes in the PSE (all $p s<0.001$ ) and not the JND (all $p s>0.1$.

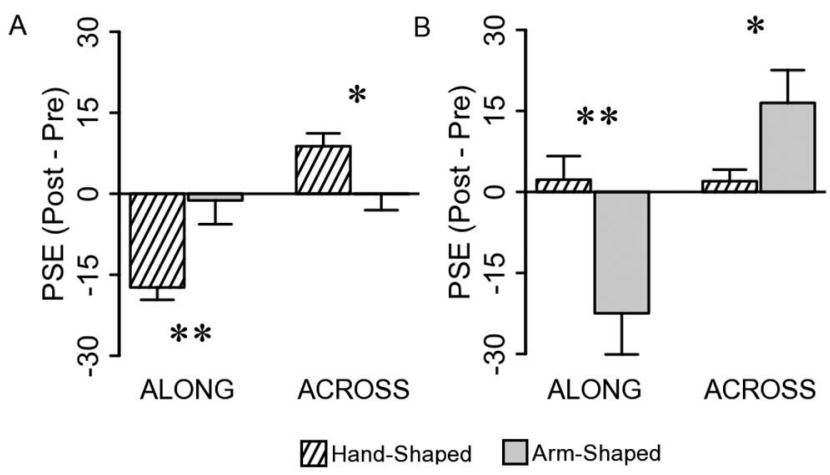

Figure 5. Tool-induced changes in the PSE for all experiments. (A) For Experiment 1, the hand was the target body part. (B) For Experiment 2, the forearm was the target body part. Dashed bars indicate the effect of the hand-shaped tool, whereas solid gray bars indicate the effect of the armshaped tool. Significant changes in tactile size perception were found in both orientations for both experiments, but only when the tool was shaped like the target body part. ${ }^{*} p<.01 .{ }^{* *} p<.001$.

\section{Experiment 2A Versus 2B}

As with Experiments $1 \mathrm{~A}$ and $1 \mathrm{~B}$, we tested whether the measured changes following the use of the arm-shaped grabber were significantly greater than that for the hand-shaped tool. We performed $2 \times 2$ ANOVA with the type of tool used (hand-shaped, arm-shaped) as a between-subjects factor, and the orientation of touch (across, along) as a within-subjects factor, with the dependent measure being the difference between the pre- and post-tooluse PSEs. Changes in tactile size perception significantly differed based on the type of tool used (Figure 5B). The interaction between tool type and orientation was significant, $F(2,22)=14.25$, $p=.001, \eta_{\mathrm{p}}^{2}=0.39$. Follow-up pairwise comparisons demonstrated a significant difference in size change between the two experiments for TDJ both across, $t(22)=2.55, p=.02, \eta^{2}=0.23$, and along, $t(22)=-3.01, p=.007, \eta^{2}=0.29$, the arm.

\section{Experiment 1 Versus 2: Shape Modulation}

Experiments $1 \mathrm{~A}$ and $2 \mathrm{~B}$ found that tool use led to opposing changes in tactile size perception across and along their respective target body parts. This suggests that the implicit body representation that underlies tactile size perception has changed its represented shape. To quantify this shape change for all four experiments (see Figure 6), we calculated a shape modulation index, which is expressed as a ratio of change between the width and length of the hand representation (Longo \& Haggard, 2010). The shape modulation index was calculated as follows:

$$
\text { Shape modulation index }=\left[\left(\frac{100+\% \text { change in hand width }}{100+\% \text { change in hand length }}\right)\right]
$$

To compare the shape modulation across all four experiments, we performed a $2 \times 2$ ANOVA with target body part (hand, arm) and tool shape (hand, arm) as between-subjects factors. Main effects for target body part, $F(1,53)=3.11, p=.08, \eta_{p}^{2}=0.06$, and tool shape, $F(1,53)=3.64, p=.06, \eta_{\mathrm{p}}^{2}=0.07$, trended but did not reach significance. Crucially, the interaction between target body part and tool shape was significant, $F(1,53)=27.65, p<$ $.0001, \eta_{p}^{2}=0.36$, indicating a relationship between the shape of the tool and the body part targeted for tool-induced representational plasticity (see Figure 6). When the tool was handshaped, there was a significantly larger shape modulation on the hand than the arm (hand $=1.33$ versus arm $=1.02$ ), $t(27)=$ $4.16, p<.0001, \eta^{2}=0.39$; the opposite pattern of results was found for the arm-shaped tool (hand $=1.04$ versus arm $=1.66$, $t(23)=3.62, p=.001, \eta^{2}=0.36$.

\section{Discussion}

We investigated whether tool-induced representational plasticity, as measured by a change in tactile size perception, was contingent upon whether the tool and the effector wielding the tool were morphologically similar. Participants wielded one of two tools, a hand-shaped tool (Figure 1A; Experiments 1A and 2A) or an arm-shaped grabber (Figure 1B; Experiments $1 \mathrm{~B}$ and $2 \mathrm{~B}$ ). Tactile size perception was tested before and after the use of the tool on the hand (Experiments 1A and 1B) or the forearm (Experiments $2 \mathrm{~A}$ and $2 \mathrm{~B}$ ). We found that using a tool led to opposing changes in tactile size perception in each orientation of the target 
A

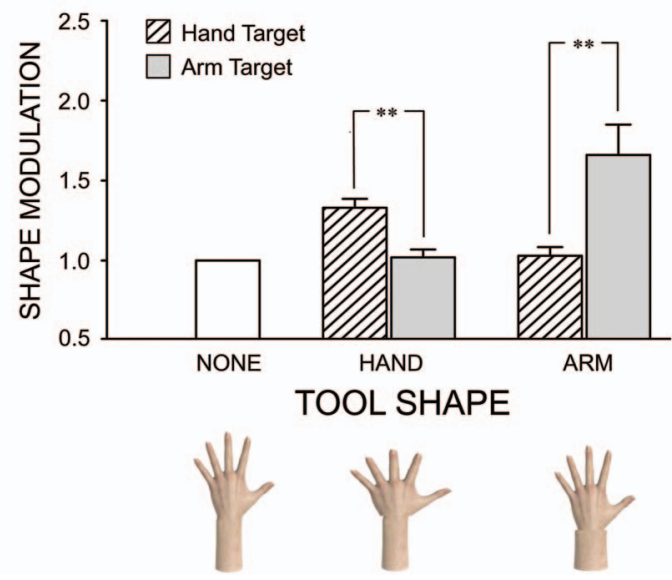

Figure 6. Shape modulation index. (A) The shape modulation index for each experiment plotted as a function of tool shape ( $x$-axis). "None" corresponds to the shape of the representation before tool use. Black bars with dashed lines correspond to experiments in which the hand was the target body part (Experiments $1 \mathrm{~A}$ and 2A). Solid gray bars correspond to the experiments in which the arm was the target body part (Experiments 1B and $2 B$ ). (B) A graphical depiction of the effect of each tool shape on the arm and hand representations. Only body parts that were shaped like the tool underwent a modulation in shape. ${ }^{* *} p<.001$. The color version of this figure appears in the online article only.

body part, namely, expansion across the limb, and compression along it. However, these findings were only found when the tool and target body part were morphologically similar (e.g., for the hand-shaped tool on the hand). No plasticity was found when the shape of the tool and the target body part did not match (e.g., for the hand-shaped tool on the arm). Effects of the tool used interacted significantly with target body part. Therefore, we demonstrated that the magnitude of plasticity to the implicit representation of an effector is constrained by whether the tool morphologically resembles said effector. Taken together with previous results emphasizing the importance of functional constraints (Bonifazi et al., 2007; Farnè et al., 2005; Farnè \& Làdavas, 2000; Holmes et al., 2007; Maravita, Clarke, et al., 2002; Maravita, Spence, et al., 2002; Serino et al., 2007; Witt et al., 2005), we conclude both function and morphology influence tool-induced representational plasticity.

\section{Representational Plasticity and Tool Morphology}

Two aspects of morphological similarity may have driven the observed representational plasticity: similarity between the structure of the tool and the body (structural similarity), and/or the similar dexterity between the tool and the body (sensorimotor similarity). Although structural similarity, which is likely to involve visual processing, had not been studied as a modulator of tool-induced representational plasticity, it has been studied for other perceptual phenomena related to body representations. For example, in the visual enhancement of touch effect, tactile spatial acuity on a body part is increased when viewing that body part, but not a non-body-shaped object (Kennett, Taylor-Clarke, \& Haggard, 2001).
Perhaps more relevant to the present findings is the shape specificity found for the RHI, in which temporally synchronous stroking by a paintbrush applied to a prosthetic hand in view and the participant's own hand that is hidden from view gives rise to the illusion that the prosthetic hand is the participant's own hand (Botvinick \& Cohen, 1998). This illusion of ownership does not occur for non-hand-shaped objects (Tsakiris et al., 2010; Tsakiris \& Haggard, 2005). Although the embodiment of a prosthetic hand depends on its structural similarity to the shape of a hand, it does not seem to depend on other aspects of visual similarity. For example, several studies have found that the presence of a RHI is not dependent upon whether subjects shared the same skin color as the prosthetic hand (Farmer, Tajadura-Jimenez, \& Tsakiris, 2012; Holmes, Snijders, \& Spence, 2006; Longo, Schuur, Kammers, Tsakiris, \& Haggard, 2009; Maister, Sebanz, Knoblich, \& Tsakiris, 2013). Similarly, although the structure of our hand-shaped tool resembles that of a hand, other visual aspects of the tool are vastly different than a human hand. Whereas the skeletal structure of the tool is similar to a hand, it lacks the volumetric properties that characterize real human hands. Further, the color of the tool (i.e., mostly white) is significantly different than the skin color of our participants. Similar differences exist between the arm-shaped grabber and the user's arm. As is the case in the RHI, the representation(s) involved in the embodiment of tools are likely underspecified and not self-specific.

There are a number of aspects of the tools other than shape that need to be considered in relation to the plasticity effects that are our primary interest. For example, in addition to shape, the tools also differed in size, which can modulate the magnitude of plasticity (Farnè et al., 2005; Sposito et al., 2012). In all of our experiments, the goals of the two tools were identical (i.e., picking up and moving balloons). However, each tool achieved this goal by altering body size in different ways. The hand-shaped tool did so by increasing hand size, while not increasing the reaching space of the arm-tool-object interactions still occurred within perihand space. Conversely, the arm-shaped grabber achieved its goal by extending the user's reaching space. It is therefore possible that the grabber's pincers were too distal in external space to modulate the user's hand representation. Effects of gross size are unlikely to fully explain our data, however, given that previous research using a short arm-shaped tool, which was of comparable size with our hand-shaped tool $(40 \mathrm{~cm})$, did find plasticity to the arm representation but not the hand representation (Cardinali et al., 2011; Cardinali, Frassinetti, et al., 2009). Nevertheless, an influence of tool size would still demonstrate that the structural morphology of the tool constrains representational plasticity, although it would not be able to speak as directly to the issue of structural and shape similarity between body and tool.

Another aspect to consider is somewhat separate from structural morphology: the sensorimotor similarity between the tool and the user's body. As described earlier, the hand-shaped tool increased the user's functional hand size, and it did so while still maintaining the dexterity of the user's own hand. The functional mapping between the user's and the tool's fingers were essentially one-toone. In contrast to the hand-shaped tool, the distal pincers of the grabber did not map directly onto the functionality of the hand and the fingers. Because of said differences in functional mapping, each tool might have influenced different body part representations, even though they were used to achieve the same goal (i.e., 
picking up and moving balloons). This process may also be influenced by differential patterns of proprioceptive feedback during the use of each tool. These aspects, like tool length, discussed previously, are broadly related to structural similarity, but need to be evaluated in more detail in future studies building on the present findings. Preliminary results appear to indicate that motor complexity of wielding the tool and proprioceptive feedback differences are unlikely to be the main driver of the representational plasticity effects observed in the present study (Miller, Longo, \& Saygin, 2013b).

Overall, in consideration of these data and previous research, we show that both structural and sensorimotor similarity can be potential modulators of representational plasticity. Of course, how the tool is used (i.e., its function) is often constrained by its physical structure, and vice versa. Tools that are shaped like hands will likely have a greater probability of being used like a hand than tools that are not hand-shaped. The structural and sensorimotor similarity of the tool are therefore intimately related, at least during typical use of the tool. Experimental work in which these factors can be teased apart can reveal the role of each aspect of morphological similarity to quantify how much each contributes to the embodiment process

\section{Mechanisms Underlying Representational Plasticity}

What are the mechanisms that underlie our finding that tool morphology constrains representational plasticity? One potential explanation comes from an influential hypothesis in the RHI literature called the body model hypothesis (Tsakiris et al., 2010). According to this hypothesis, embodiment requires a match-totemplate process in which the morphology of the object is compared against a stored representation of body structure, and accordingly, representational plasticity would be constrained by whether an external object is structurally similar to the body. Although several recent findings have called into question the explanatory limits of the body model hypothesis for the RHI (e.g., participants can be made to experience ownership over three arms; Ehrsson, 2009; Guterstam, Petkova, \& Ehrsson, 2011; but see de Vignemont \& Farnè, 2010 and Folegatti, Farnè, Salemme, \& de Vignemont, 2012), or even a volume of empty space (Guterstam, Gentile, \& Ehrsson, 2013), our findings are nevertheless the first to implicate a match-to-template process in tool-induced plasticity. Further, although the template discussed in the RHI is often a visual one, our results leave open the possibility that tools access a sensorimotor template when targeting specific body parts during embodiment. This sensorimotor body template may explain how we can use certain tools despite them having little to no visual resemblance to the body (see the Body Representations and Tool Embodiment section for a more thorough discussion of this point).

We can gain more insight into the observed representational plasticity by considering possible neural mechanisms underlying tactile size perception. Whereas tactile size perception is dependent upon an implicit representation of body morphology (Longo et al., 2010), likely in posterior parietal cortex (PPC), it has also been tied to the geometry of receptive fields (RFs) in primary somatosensory cortex (SI; Longo \& Haggard, 2011). Visual body information from the PPC - which may store the visual body template discussed in the previous paragraph (Konen \& Haggard, 2012) - can modulate levels of intracortical inhibition (Cardini,
Longo, \& Haggard, 2011), altering SI RF geometry (Haggard, Christakou, \& Serino, 2007). Changes to intracortical inhibition often differentially affect different axes of the RF, leading to anisotropic changes in its shape (Alloway, Rosenthal, \& Burton, 1989). Furthermore, neurophysiology studies have found that tool use modulates neuronal processing in both primary somatosensory cortex and posterior parietal cortex in the macaque monkey (Iriki et al., 1996; Quallo et al., 2009). We thus suggest that top-down signals from the PPC during tool use may cause anisotropic changes in SI RF geometry, leading to the observed plasticity to tactile size perception.

\section{The Relationship Between Plasticity and Represented Shape}

Following use of the hand-shaped tool, did the underlying hand representation become short and squat, or skinny and long? The answer to this question is dependent upon on how we conceptualize the relationship between the size of an implicit limb representation and the perceived size of touch on that limb. Two opposing views have been discussed in the literature, which we summarize in Figure 7. In one view, there is an inverse relationship between representational size and perceived tactile size. As the size of the representation increases, two points of touch on the somatosensory homunculus in SI are remapped onto smaller ana-
A

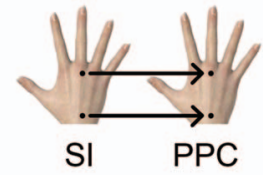

B

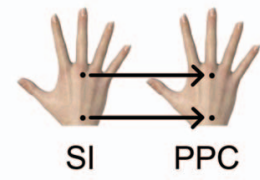

C

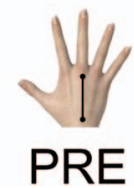

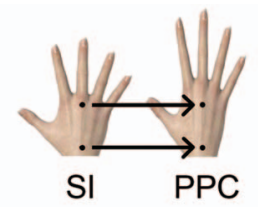
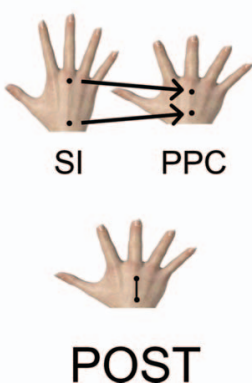

Figure 7. Relationship between tactile size perception and body representations. Tactile size perception may have an inverse (A) or proportional (B) relationship with the dimensions of an implicit representation of body morphology. In the pre-tool-use condition, two points of touch in the somatosensory homunculus of the hand in primary somatosensory cortex (SI; the hand on the left in each pairing) are mapped onto congruent locations on the implicit hand representation in the posterior parietal cortex (PPC; the hand on the right in each pairing). According to the inverse model (A), the decrease in tactile size perception observed in the posttool-use condition would be the result of mapping two points of touch onto a smaller anatomical location on the implicit representation because of an increase in its size. Conversely, the proportional model (B) states that the decrease in tactile size perception is because of a corresponding decrease in the size of the implicit representation. (C) The perceived tactile size, represented by a line between the two points of touch, is equivalent for both models pre- and post-tool use. The color version of this figure appears in the online article only. 
tomical locations on the implicit body representation in the PPC, leading them to be perceived as closer together. In the other view, there is a proportional relationship between representational size and perceived tactile size. As the size of the representation decreases, so does the perceived distance between two points of touch. This view is espoused by Longo and Haggard (2011), who propose that the dimensions of implicit body representations are dependent upon the geometry of receptive fields in SI. In this view, tactile size perception is inextricably linked to the dimensions of the implicit body representation.

A recent study (Canzoneri et al., 2013) found that using a long rake led to a decrease in tactile size perception proximodistally on the arm (consistent with our results) and a concurrent distal shift in tactile localization (consistent with Cardinali, Frassinetti, et al., 2009). Although these findings may lend empirical support for the inverse view, the lack of correlation between the magnitude of plasticity in the two tasks suggests caution in making a straightforward interpretation. However, several studies using different methods have found evidence that tool use increases the represented size of body parts. For example, a tool-user's reaching kinematics are modulated in the direction expected if the length of their arm representation had increased (Cardinali et al., 2012; Cardinali, Frassinetti, et al., 2009). Further, there is a distal shift in the location where participants bisect their arms after tool use, suggesting that their arm is represented as longer post-tool use (Sposito et al., 2012). Although there are likely differences between representations underlying kinematics and tactile perception (see the Body Representations and Tool Embodiment section), these studies at least lend indirect support to the inverse view.

Although our data do not allow us to definitively resolve the issue, we are inclined to interpret our results within the framework of the proportional model. Under this view, the implicit hand representation would become short and squat following use of the hand-shaped tool. Support for the proportional view comes from studies showing a positive relationship between perceived tactile size and cortical magnification (Gibson \& Craig, 2005; TaylorClarke et al., 2004). Increases in tactile size perception have been observed when changes in visual (Taylor-Clarke et al., 2004), auditory (Tajadura-Jiménez et al., 2012), or proprioceptive (de Vignemont et al., 2005) input led to the perception that the limb had increased in size. Further, consistent with findings from the TDJ (Longo \& Haggard, 2011), a paradigm requiring participants to explicitly locate landmarks on their hand (psychomorphometric paradigm) found that the dorsal surface of the hand is perceived as short and squat (Longo \& Haggard, 2010). The correspondence between findings from the psychomorphometric paradigm and the TDJ suggest that the two tasks rely on the same implicit body representation. Although this needs to be verified in future studies, we observed the same pattern of tool-induced shape plasticity for an explicit, visual body representation in a preliminary study (Miller, Longo, \& Saygin, 2013a).

Knowing the relationship between tactile size perception and body representation shape would help resolve an apparent conflict between our findings and previous research. How can tool use lead to an apparent lengthening of the arm representation (Cardinali et al., 2011; Cardinali, Frassinetti, et al., 2009; Sposito et al., 2012) and compression in tactile size perception? There is no conflict if the inverse view is correct. However, if the proportional view is correct, a discrepancy exists between findings using the TDJ and other paradigms, such as tactile localization (Canzoneri et al., 2013; Cardinali et al., 2011; Cardinali, Frassinetti, et al., 2009). One potential explanation for this discrepancy comes from the psychophysics literature on tactile localization. Tactile localization is more accurate on skin surfaces with high spatial acuity (Cody, Garside, Lloyd, \& Poliakoff, 2008). Viewing the body, which increases tactile spatial acuity (Kennett et al., 2001), could also increase the speed of tactile localization (Press, Taylor-Clarke, Kennett, \& Haggard, 2004) and compress tactile size perception (Longo \& Sadibolova, 2013). Examination of Figure 5 in Canzoneri and colleagues (2013) reveals that the perceptual distalization of touch following tool use reflects a decrease in spatial localization error. Although the relationship between tactile size perception and localization is complicated and needs to be further specified, it is possible that both compression and distalization reflect similar tool-induced modulations to the mechanisms underlying tactile spatial perception.

\section{Body Representations and Tool Embodiment}

How to conceptualize body representations has been a major topic of debate in psychology for decades. To date, there is little agreement between researchers, both scientists and philosophers, on how many representations of the body there are and their functional properties. On the one hand, researchers hope to capture the complexities of what it is like to have a body (Longo \& Haggard, 2012b), whereas, on the other hand, not inventing a new body representation for every novel task (Kammers, Mulder, de Vignemont, \& Dijkerman, 2010).

Perhaps the most common taxonomy divides representations of the body into those for action (body schema) and those for perception (body image; de Vignemont, 2010; Dijkerman \& de Haan, 2007; Gallagher, 1986). The body schema is conceptualized as an online sensorimotor representation of the body that is continually updated as the body's posture changes. The body image, in contrast, is conceptualized as an offline representation that structures first-person body perception in the visual and somatosensory modalities. Empirical studies of both neurological patients (Paillard, 1999; Rossetti, Rode, \& Boisson, 1995) and healthy participants (e.g., Kammers, de Vignemont, Verhagen, \& Dijkerman, 2009; Kammers, van der Ham, \& Dijkerman, 2006) have lent support to this distinction. Other researchers have sought to elaborate on this dyadic view of the body. For example, Longo and Haggard (2010, 2012a) have proposed a representation that they call the body model, which is distinct from both the body schema and body image. Under this view, the body image is best conceived of as a visual representation of body shape, whereas the body model is primarily somatosensory (Longo et al., 2010).

As of now, we have used the somewhat generic term implicit body representation when referring to the representation(s) underlying tactile perception. Although our results do not directly speak to how to best divide and enumerate body representations, what levels of body-related processing are affected by tool embodiment is an important empirical question. A discussion on how to conceptualize our results in terms of a specific body representation is therefore warranted.

Several theorists have claimed that tasks measuring tactile perception tap into a tactile body image (de Vignemont, 2010; Dijk- 
erman \& de Haan, 2007). However, including visual and somatosensory measures of body shape under the representation "body image" ignores differences in how accurately judgments in each modality reflect actual body shape-for example, somatosensory judgments appear to retain homuncular distortions (Longo \& Haggard, 2010, 2011), whereas visual judgments are more veridical (Longo \& Haggard, 2012a). We believe that this important difference is best captured by a distinction between the body image and body model. Given the distortions observed in tool-induced modulations to tactile perception (this study; Canzoneri et al., 2013; Cardinali et al., 2011; Cardinali, Frassinetti, et al., 2009), these effects might be thought of as changes to a primarily tactile body model.

The necessary and sufficient conditions of tool embodiment likely differ between body representations. Our data suggests that representational plasticity to a body part within the body model is crucially dependent upon whether the tool is morphologically similar to that body part. This appears to be the case for the body schema as well. Cardinali, Frassinetti, and colleagues (2009) found that using an arm-shaped tool led a change to the arm-but not the hand-representation in the body schema. However, if representational plasticity is a necessary precondition for the ability to use a tool, template matching proposes a problem, as we are able to use objects whose shape does not clearly match any body part template (e.g., corkscrews). This problem may be remedied if the necessity of tool shape is dependent upon the type of representation undergoing embodiment. One important functional characteristic that is shared between the body model and body schema is that they are both about the body itself, and are inextricably linked to its shape. Peripersonal space, on the other hand, is not specifically about the body proper (Cardinali, Brozzoli, \& Farnè, 2009), but is instead about the space between the body and objects within its current action space (Brozzoli, Ehrsson, \& Farnè, 2013). Embodiment of tools into peripersonal space may therefore depend more upon whether an object modifies the boundaries of the user's action space and less upon whether that object has a close physical resemblance to the body. Indeed, tools baring no resemblance to a hand (e.g., sticks) have been shown to modify the boundaries where multisensory interactions between touch on the hand and visual objects can take place (Farnè et al., 2005; Farnè \& Làdavas, 2000; Maravita, Spence, et al., 2002). The relative indifference to the tool's shape of some representations related to the body would allow for our ability to embody and use tools that bear little to no resemblance to our own bodies.

These differences in body representations have important implications for the design of prosthetic limbs. The goal of prosthetic use-incorporation into the sense of bodily self (Murray, 2004) - is aided by the usability of the prostheses (Nico, Daprati, Rigal, Parsons, \& Sirigu, 2004), underscoring the importance of sensorimotor processes in embodiment. Our findings, on the other hand, demonstrate the importance of structural similarity in representational plasticity, especially for body representations that are specifically body-oriented (e.g., body schema and body model). This suggests that cosmetic aspects of prosthetic design may further aid in its embodiment into multiple levels of body representation, leading to less cases of prosthetic rejection.

\section{Conclusion}

We found that functional use of a tool led to representational plasticity on two body parts, the hand and arm, but only when the tool was shaped like that body part. These results demonstrate that the morphology of a tool constrains representational plasticity. Morphological similarity has previously been shown to constrain the embodiment of rubber hands, suggesting that sensitivity to shape may reflect a widespread property of body representations and embodiment. Our results indicate that both function and morphological similarity are necessary for the embodiment of tools, factors that should be taken into consideration for prosthetic design.

\section{References}

Alloway, K. D., Rosenthal, P., \& Burton, H. (1989). Quantitative measurements of receptive field changes during antagonism of gabaergic transmission in primary somatosensory cortex of cats. Experimental Brain Research, 78, 514-532. doi:10.1007/BF00230239

Bonifazi, S., Farnè, A., Rinaldesi, L., \& Làdavas, E. (2007). Dynamic size-change of peri-hand space through tool-use: Spatial extension or shift of the multi-sensory area. Journal of Neuropsychology, 1, 101-114. doi:10.1348/174866407X180846

Botvinick, M., \& Cohen, J. (1998). Rubber hands "feel" touch that eyes see. Nature, 391, 756. doi:10.1038/35784

Brozzoli, C., Ehrsson, H. H., \& Farnè, A. (2013). Multisensory representation of the space near the hand: From perception to action and interindividual interactions. Neuroscientist, 20, 122-135. doi:10.1177/1073858413511153

Butler, S. (1872). Erewhon; or, over the range. London, UK: Trübner.

Canzoneri, E., Ubaldi, S., Rastelli, V., Finisguerra, A., Bassolino, M., \& Serino, A. (2013). Tool-use reshapes the boundaries of body and peripersonal space representations. Experimental Brain Research, 228, 25-42.

Cardinali, L., Brozzoli, C., \& Farnè, A. (2009). Peripersonal space and body schema: Two labels for the same concept? Brain Topography, 21, 252-260. doi:10.1007/s10548-009-0092-7

Cardinali, L., Brozzoli, C., Urquizar, C., Salemme, R., Roy, A. C., \& Farnè, A. (2011). When action is not enough: Tool-use reveals tactiledependent access to body schema. Neuropsychologia, 49, 3750-3757. doi:10.1016/j.neuropsychologia.2011.09.033

Cardinali, L., Frassinetti, F., Brozzoli, C., Urquizar, C., Roy, A. C., \& Farnè, A. (2009). Tool-use induces morphological updating of the body schema. Current Biology, 19, R478-R479. doi:10.1016/j.cub.2009.05 .009

Cardinali, L., Jacobs, S., Brozzoli, C., Frassinetti, F., Roy, A., \& Farnè, A. (2012). Grab an object with a tool and change your body: Tool-usedependent changes of body representation for action. Experimental Brain Research, 218, 259-271. doi:10.1007/s00221-012-3028-5

Cardini, F., Longo, M. R., \& Haggard, P. (2011). Vision of the body modulates somatosensory intracortical inhibition. Cerebral Cortex, 21 2014-2022. doi:10.1093/cercor/bhq267bhq267

Cody, F. W., Garside, R., Lloyd, D., \& Poliakoff, E. (2008). Tactile spatial acuity varies with site and axis in the human upper limb. Neuroscience Letters, 433, 103-108. doi:10.1016/j.neulet.2007.12.054

Costantini, M., Ambrosini, E., Sinigaglia, C., \& Gallese, V. (2011). Tooluse observation makes far objects ready-to-hand. Neuropsychologia, 49, 2658-2663. doi:10.1016/j.neuropsychologia.2011.05.013

de Vignemont, F. (2010). Body schema and body image-pros and cons. Neuropsychologia, 48, 669-680. doi:10.1016/j.neuropsychologia.2009 .09 .022 
de Vignemont, F., Ehrsson, H. H., \& Haggard, P. (2005). Bodily illusions modulate tactile perception. Current Biology, 15, 1286-1290. doi:10.1016/ j.cub.2005.06.067

de Vignemont, F., \& Farnè, A. (2010). Widening the body to rubber hands and tools: What's the difference? La revue de neuropsychologie, 2, 203-211.

Dijkerman, H. C., \& de Haan, E. H. F. (2007). Somatosensory processes subserving perception and action. Behavioral and Brain Sciences, 30, 189-201; discussion 201-239. doi:10.1017/S0140525x07001392

Ehrsson, H. H. (2009). How many arms make a pair? Perceptual illusion of having an additional limb. Perception, 38, 310-312. doi:10.1068/p6304

Farmer, H., Tajadura-Jimenez, A., \& Tsakiris, M. (2012). Beyond the colour of my skin: How skin colour affects the sense of body-ownership. Consciousness and Cognition: An International Journal, 21, 12421256. doi:10.1016/j.concog.2012.04.011

Farnè, A., Iriki, A., \& Làdavas, E. (2005). Shaping multisensory actionspace with tools: Evidence from patients with cross-modal extinction. Neuropsychologia, 43, 238-248. doi:10.1016/j.neuropsychologia.2004 .11 .010

Farnè, A., \& Làdavas, E. (2000). Dynamic size-change of hand peripersonal space following tool use. NeuroReport: For Rapid Communication of Neuroscience Research, 11, 1645-1649. doi:10.1097/00001756200006050-00010

Folegatti, A., Farnè, A., Salemme, R., \& de Vignemont, F. (2012). The rubber hand illusion: Two's a company, but three's a crowd. Consciousness and Cognition: An International Journal, 21, 799-812. doi:10.1016/j.concog .2012 .02 .008

Gallagher, S. (1986). Body image and body schema: A conceptual clarification. Journal of Mind and Behavior, 7, 541-554.

Gibson, G. O., \& Craig, J. C. (2005). Tactile spatial sensitivity and anisotropy. Perception \& Psychophysics, 67, 1061-1079. doi:10.3758/ BF03193632

Guterstam, A., Gentile, G., \& Ehrsson, H. H. (2013). The invisible hand illusion: Multisensory integration leads to the embodiment of a discrete volume of empty space. Journal of Cognitive Neuroscience, 25, $1078-$ 1099. doi:10.1162/jocn_a_00393

Guterstam, A., Petkova, V. I., \& Ehrsson, H. H. (2011). The illusion of owning a third arm. PLoS ONE, 6, e17208. doi:10.1371/journal.pone .0017208

Haans, A., IJsselsteijn, W. A., \& de Kort, Y. A. (2008). The effect of similarities in skin texture and hand shape on perceived ownership of a fake limb. Body Image, 5, 389-394. doi:10.1016/j.bodyim.2008.04.003

Haggard, P., Christakou, A., \& Serino, A. (2007). Viewing the body modulates tactile receptive fields. Experimental Brain Research, 180, 187-193. doi:10.1007/s00221-007-0971-7

Holmes, N. P., Calvert, G. A., \& Spence, C. (2007). Tool use changes multisensory interactions in seconds: Evidence from the crossmodal congruency task. Experimental Brain Research, 183, 465-476. doi: 10.1007/s00221-007-1060-7

Holmes, N. P., Snijders, H. J., \& Spence, C. (2006). Reaching with alien limbs: Visual exposure to prosthetic hands in a mirror biases proprioception without accompanying illusions of ownership. Perception \& Psychophysics, 68, 685-701. doi:10.3758/BF03208768

Iriki, A., Tanaka, M., \& Iwamura, Y. (1996). Coding of modified body schema during tool use by macaque postcentral neurones. NeuroReport, 7, 2325-2330. doi:10.1097/00001756-199610020-00010

Kammers, M. P., de Vignemont, F., Verhagen, L., \& Dijkerman, H. C. (2009). The rubber hand illusion in action. Neuropsychologia, 47, 204211. doi:10.1016/j.neuropsychologia.2008.07.028

Kammers, M. P., Mulder, J., de Vignemont, F., \& Dijkerman, H. C. (2010). The weight of representing the body: Addressing the potentially indefinite number of body representations in healthy individuals. Experimental Brain Research, 204, 333-342. doi:10.1007/s00221-009-2009-9
Kammers, M. P., van der Ham, I. J., \& Dijkerman, H. C. (2006). Dissociating body representations in healthy individuals: Differential effects of a kinaesthetic illusion on perception and action. Neuropsychologia, 44, 2430-2436. doi:10.1016/j.neuropsychologia.2006.04.009

Kao, K. L., \& Goodale, M. A. (2009). Enhanced detection of visual targets on the hand and familiar tools. Neuropsychologia, 47, 2454-2463. doi:10.1016/j.neuropsychologia.2009.04.016

Kennett, S., Taylor-Clarke, M., \& Haggard, P. (2001). Noninformative vision improves the spatial resolution of touch in humans. Current Biology, 11, 1188-1191

Konen, C. S., \& Haggard, P. (2012). Multisensory parietal cortex contributes to visual enhancement of touch in humans: A single-pulse TMS study. Cerebral Cortex, doi:10.1093/cercor/bhs331

Longo, M. R., Azanon, E., \& Haggard, P. (2010). More than skin deep: Body representation beyond primary somatosensory cortex. Neuropsychologia, 48, 655-668. doi:10.1016/j.neuropsychologia.2009.08.022

Longo, M. R., \& Haggard, P. (2010). An implicit body representation underlying human position sense. PNAS Proceedings of the National Academy of Sciences of the United States of America, 107, 1172711732. doi:10.1073/pnas.1003483107

Longo, M. R., \& Haggard, P. (2011). Weber's illusion and body shape Anisotropy of tactile size perception on the hand. Journal of Experimental Psychology: Human Perception and Performance, 37, 720-726. doi:10.1037/a0021921

Longo, M. R., \& Haggard, P. (2012a). Implicit body representations and the conscious body image. Acta Psychologica, 141, 164-168. doi:10.1016/j actpsy.2012.07.015

Longo, M. R., \& Haggard, P. (2012b). What is it like to have a body? Current Directions in Psychological Science, 21, 140-145. doi:10.1177/ 0963721411434982

Longo, M. R., \& Sadibolova, R. (2013). Seeing the body distorts tactile size perception. Cognition, 126, 475-481. doi:10.1016/j.cognition.2012 .11 .013

Longo, M. R., Schuur, F., Kammers, M. P., Tsakiris, M., \& Haggard, P. (2009). Self-awareness and the body image. Acta Psychologica, 132, 166-172. doi:10.1016/j.actpsy.2009.02.003

Maister, L., Sebanz, N., Knoblich, G., \& Tsakiris, M. (2013). Experiencing ownership over a dark-skinned body reduces implicit racial bias. Cognition, 128, 170-178. doi:10.1016/j.cognition.2013.04.002

Maravita, A., Clarke, K., Husain, M., \& Driver, J. (2002). Active tool use with the contralesional hand can reduce cross-modal extinction of touch on that hand. Neurocase, 8, 411-416. doi:10.1093/neucas/8.6.411

Maravita, A., \& Iriki, A. (2004). Tools for the body (schema). Trends in Cognitive Sciences, 8, 79-86. doi:10.1016/j.tics.2003.12.008

Maravita, A., Spence, C., Kennett, S., \& Driver, J. (2002). Tool-use changes multimodal spatial interactions between vision and touch in normal humans. Cognition, 83, B25-B34. doi:10.1016/S0010-0277(02)00003-3

Miller, L. E., Longo, M. R., \& Saygin, A. P. (2013a). Tool use modulates both conscious and unconscious representations of body shape. Paper presented at the Association for the Scientific Study of Consciousness, San Diego, CA.

Miller, L. E., Longo, M. R., \& Saygin, A. P. (2013b). Visual feedback constrains representational plasticity following tool use. Paper presented at the Society for Neuroscience, San Diego, CA.

Murray, C. D. (2004). An interpretative phenomenological analysis of the embodiment of artificial limbs. Disability and Rehabilitation, 26, $963-$ 973. doi:10.1080/09638280410001696764

Nico, D., Daprati, E., Rigal, F., Parsons, L., \& Sirigu, A. (2004). Left and right hand recognition in upper limb amputees. Brain, 127, 120-132. doi:10.1093/brain/awh006

Paillard, J. (1999). Body schema and body image: A double dissociation in deafferented patients. In G. N. Gantchev, S. Mori \& J. Massion (Eds.), Motor Control Today and Tomorrow (pp. 197-214). Sofia, Bulgaria: Academic Publishing House. 
Press, C., Taylor-Clarke, M., Kennett, S., \& Haggard, P. (2004). Visual enhancement of touch in spatial body representation. Experimental Brain Research, 154, 238-245. doi:10.1007/s00221-003-1651-x

Prins, N., \& Kingdom, F. A. A. (2009). Palamedes: Matlab routines for analyzing psychophysical data. Retrived from http://www .palamedestoolbox.org

Quallo, M. M., Price, C. J., Ueno, K., Asamizuya, T., Cheng, K., Lemon, R. N., \& Iriki, A. (2009). Gray and white matter changes associated with tool-use learning in macaque monkeys. PNAS Proceedings of the National Academy of Sciences of the United States of America, 106, 18379-18384. doi:10.1073/pnas.0909751106

Rossetti, Y., Rode, G., \& Boisson, D. (1995). Implicit processing of somaesthetic information: A dissociation between where and how? Neuroreport: An International Journal for the Rapid Communication of Research in Neuroscience, 6, 506-510. doi:10.1097/00001756199502000-00025

Serino, A., Bassolino, M., Farnè, A., \& Làdavas, E. (2007). Extended multisensory space in blind cane users. Psychological Science, 18, 642-648. doi:10.1111/j.1467-9280.2007.01952.x

Sposito, A., Bolognini, N., Vallar, G., \& Maravita, A. (2012). Extension of perceived arm length following tool-use: Clues to plasticity of body metrics. Neuropsychologia, 50, 2187-2194. doi:10.1016/j.neuropsychologia.2012 .05 .022

Tajadura-Jiménez, A., Väljamäe, A., Toshima, I., Kimura, T., Tsakiris, M., \& Kitagawa, N. (2012). Action sounds recalibrate perceived tactile distance. Current Biology, 22, R516-R517. doi:10.1016/j.cub.2012.04.028

Taylor-Clarke, M., Jacobsen, P., \& Haggard, P. (2004). Keeping the world a constant size: Object constancy in human touch. Nature Neuroscience, 7, 219-220. doi:10.1038/nn1199
Tsakiris, M., Carpenter, L., James, D., \& Fotopoulou, A. (2010). Hands only illusion: Multisensory integration elicits sense of ownership for body parts but not for non-corporeal objects. Experimental Brain Research, 204, 343-352. doi:10.1007/s00221-009-2039-3

Tsakiris, M., Costantini, M., \& Haggard, P. (2008). The role of the right temporo-parietal junction in maintaining a coherent sense of one's body Neuropsychologia, 46, 3014-3018. doi:10.1016/j.neuropsychologia .2008.06.004

Tsakiris, M., \& Haggard, P. (2005). The rubber hand illusion revisited: Visuotactile integration and self-attribution. Journal of Experimental Psychology: Human Perception and Performance, 31, 80-91. doi: 10.1037/0096-1523.31.1.80

Wichmann, F. A., \& Hill, N. J. (2001a). The psychometric function: I. Fitting, sampling, and goodness of fit. Perception \& Psychophysics, 63 , 1293-1313. doi:10.3758/BF03194544

Wichmann, F. A., \& Hill, N. J. (2001b). The psychometric function: Ii. Bootstrap-based confidence intervals and sampling. Perception \& Psychophysics, 63, 1314-1329. doi:10.3758/BF03194545

Witt, J. K., Proffitt, D. R., \& Epstein, W. (2005). Tool use affects perceived distance, but only when you intend to use it. Journal of Experimental Psychology: Human Perception and Performance, 31, 880-888. doi: 10.1037/0096-1523.31.5.880

Received July 20, 2013

Revision received May 27, 2014

Accepted May 30, 2014 